\title{
GaN GROWTH BY REMOTE PLASMA MOCVD: CHEMISTRY AND KINETICS BY REAL TIME ELLIPSOMETRY
}

M. Losurdo, P. Capezzuto and G. Bruno

Plasma Chemistry Research Center-CNR, via Orabona 4- 70126 Bari, Italy, cscpml18@area.ba.cnr.it

Cite this article as: MRS Internet J. Nitride Semicond. Res. 4S1, G3.12(1999)

\begin{abstract}
Cubic and hexagonal GaN layers have been grown on GaAs (001) and $\alpha-\mathrm{Al}_{2} \mathrm{O}_{3}(0001)$ substrates, respectively, by remote plasma metalorganic chemical vapor deposition (RPMOCVD). In situ spectroscopic ellipsometry is used to monitor in real time the chemistry and kinetics of the $\mathrm{GaN}$ growth. The subtrate/GaN interface formation is highlighted and the effect of the substrate plasma nitridation on the initial growth stage is discussed.
\end{abstract}

\section{INTRODUCTION}

There have been numerous recent publications on the effects of the two growth steps, i.e., substrate nitridation followed by the growth of a low temperature nucleation layer, upon the material properties of $\mathrm{GaN}$ epilayers grown by both molecular beam epitaxy (MBE) and metalorganic chemical vapor deposition (MOCVD) [1,2]. And, it has been shown that slight differences in the substrate nitridation and/or the growth of the $\mathrm{GaN}$ buffer layer prior to $\mathrm{GaN}$ epigrowth results in different $\mathrm{GaN}$ growth mechanisms [3,4]. Although the number of experimental studies conducted to date is already significant, it is not yet possible to establish an all-embracing theory of the influence of the surface properties upon the GaN growth chemistry and kinetics. And, it is widely recognized that a deeper understanding of the fundamental surface kinetics governing nitride growth is necessary to move from the "GaN art" to the "GaN science". While the investigation of the GaN growth mechanism is ongoing for MBE systems, where a variety of in situ diagnostic techniques can be used, such as reflection high energy electron diffraction (RHEED), low energy electron diffraction (LEED), X-ray photoelectron spectroscopy (XPS), some difficulties are encountered for the MOCVD process, where high pressure and reactive environment prevent from the use of the above techniques.

In this paper, the remote plasma MOCVD (RP-MOCVD) of GaN buffer layers grown on both $\alpha-\mathrm{Al}_{2} \mathrm{O}_{3}$ and $\mathrm{GaAs}$ substrates, also pre-nitrided by $\mathrm{N}_{2}$ plasmas, is discussed. Particular emphasis is placed on the in situ real time control of the growing surface and of the substrate/GaN interface by spectroscopic ellipsometry (SE), which is a non-invasive and nonintrusive optical technique compatible with the MOCVD environment. Ellipsometry data are used to depict the chemistry and kinetics of the initial stage of the GaN growth.

\section{EXPERIMENT}

The deposition system used in this study is the remote plasma MOCVD apparatus specifically designed for the growth and treatment of III-V materials [5] at reduced pressure $(0.1$ - 10 Torr) and reduced temperature. A unique feature of this apparatus is the presence of an in situ phase modulated ellipsometer (UVISEL-ISA Jobin-Yvon) compatible with the reactive environment of MOCVD reactors and, hence, able to monitor in real time the chemistry and kinetics of surface modifications with a sub-monolayer resolution. The substrates used for the growth were GaAs (001) and $\alpha-\mathrm{Al}_{2} \mathrm{O}_{3}$ (0001). The remote r.f. (13.56 MHz) plasma source was 
used to produce $\mathrm{H}$-atoms for the substrates cleaning, and $\mathrm{N}$-atoms for the nitridation process and $\mathrm{GaN}$ growth. The $\mathrm{H}_{2}$ remote plasma cleaning was operated at $\mathrm{P}=1$ Torr, r.f. power $=60$ Watt and at a surface temperature of $250^{\circ} \mathrm{C}$ and $350^{\circ} \mathrm{C}$ for $\mathrm{GaAs}(001)$ and $\alpha-\mathrm{Al}_{2} \mathrm{O}_{3}(0001)$, respectively. The subsequent nitridation by $\mathrm{N}_{2}-\mathrm{H}_{2}\left(3 \%\right.$ in $\left.\mathrm{H}_{2}\right)$ plasmas was operated at a pressure of 0.2 Torr, a r.f. power of 200 Watt and at a temperature of $250^{\circ} \mathrm{C}$ and $800^{\circ} \mathrm{C}$ for $\mathrm{GaAs}$ and $\alpha-\mathrm{Al}_{2} \mathrm{O}_{3}$, respectively. $\mathrm{GaN}$ layers were grown at $600^{\circ} \mathrm{C}$ by trymethylgallium and $\mathrm{N}_{2} / \mathrm{H}_{2}$ $(=1000 / 3 \mathrm{sccm})$ plasma at a pressure of 1 Torr and a r.f. power of $200 \mathrm{~W}$. Single wavelength ellipsometry (SWE) was used, in terms of the ellipsometric angles $\Psi$ and $\Delta[6]$, to monitor in real time the surface kinetics, and, at the growth end, ellipsometric spectra (SE) of the pseudodielectric function, $\langle\varepsilon\rangle=\left\langle\varepsilon_{1}\right\rangle+\mathrm{i}\left\langle\varepsilon_{2}\right\rangle$, were acquired in the energy range $1.5-5.5 \mathrm{eV}$. In order to determine the chemical and optical properties of surfaces, ellipsometric data were modeled by optical models based on the Bruggeman effective-medium approximation (BEMA) [6] and references for the used dielectric functions are given in ref. [7]. Optical emission spectroscopy (OES) was used to control the $\mathrm{N}$-atom density interacting with the growth surface. Ex situ atomic force microscopy (AFM) and X-ray diffraction (XRD) measurements were also performed to validate the ellipsometric approach.

\section{RESULTS AND DISCUSSION}

Figure 1 shows typical SWE real time $(\Psi, \Delta)$ trajectories recorded at $3 \mathrm{eV}$ during $\mathrm{GaN}$ growth on both $\alpha-\mathrm{Al}_{2} \mathrm{O}_{3}$ (0001) and $\mathrm{GaAs}$ (001) substrates previously nitrided. An easily provided information is the $\mathrm{GaN}$ layer thickness which is controlled in real time.
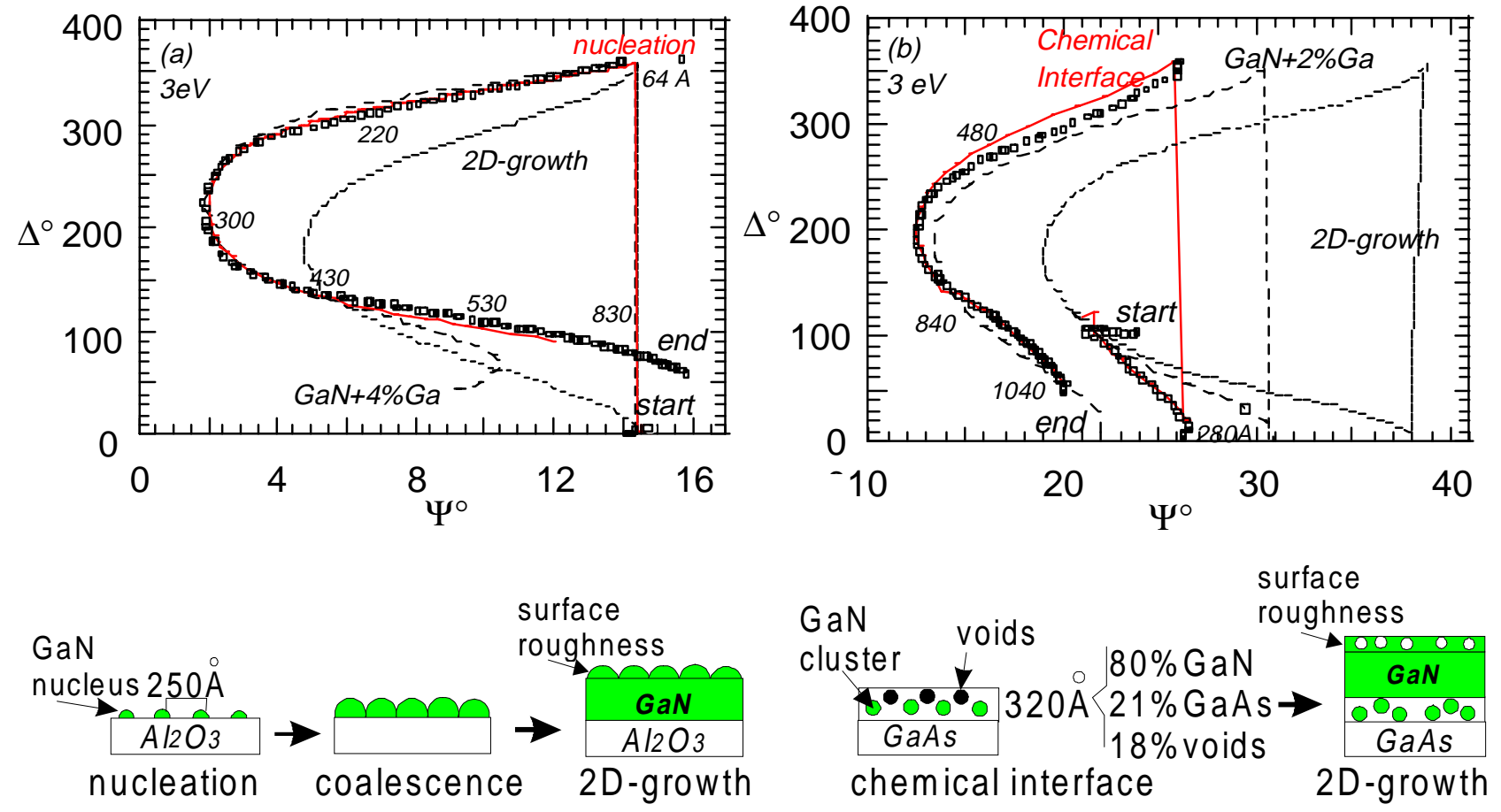

FIGURE 1. Time evolution of the optical response in the $(\Psi, \Delta)$ space at $3 \mathrm{eV}$ during $\mathrm{GaN}$ growth on (a) $\alpha-\mathrm{Al}_{2} \mathrm{O}_{3}$ and (b) GaAs. Points are for experimental data; full line is for the best-fit model which is, for $\mathrm{GaN}$ on $\alpha-\mathrm{Al}_{2} \mathrm{O}_{3}$, nucleation followed by $2 \mathrm{D}$-homogeneous growth and, for GaN on GaAs, chemical interface appearance followed by 2D-homogeneous growth. Simulated trajectories by different models (see text) are also reported for comparison. 
For a better understanding of the $(\Psi, \Delta)$ data, it has to be considered that at $3 \mathrm{eV}$ (below energy gap value, where the GaN absorption is almost zero) a closed and cyclical trajectory should be recorded for a well ordered and pure GaN layer. Thus, deviations from this ideal cyclical curve are indicative of non-homogeneous growth dynamics and/or defected absorbing layers. For the $(\Psi, \Delta)$ trajectories simulation, different models have been considered such as the 2Dhomogeneous growth, the nucleation followed by homogeneous growth and the stoichiometry deviation in terms of Ga enrichment. Among these models, only the "nucleation followed by homogeneous growth with roughness" model fits the whole experimental trajectory in fig. 1(a). Specifically, the growth process of the $\mathrm{GaN}$ epilayer on $\alpha-\mathrm{Al}_{2} \mathrm{O}_{3}$ can be distinguished into two regimes, i.e., an initial hemispherical nucleation regime followed by the GaN bulk growth (see scheme in fig. 1a). The nucleation density on the starting surface produces a mean separation of $\sim 250 \AA$ between nuclei, whose radius increases according to the the law $R(t)=1.8(\AA / \mathrm{sec}) \cdot \mathrm{t}$ until they come into contact. As soon as the complete nuclei coalescence is obtained, the homogeneous GaN growth starts with a steady-state growth rate of about $1 \AA / \mathrm{sec}$ and a gradual roughening of the growing surface also confirmed by SE modeling (see below) and AFM measurements.

For the GaN growth on GaAs (fig. 1b), the regression analysis fit evidences the appearance of a chemical interface between the substrate and the GaN film. For the interface formation regime, free fit parameters include the volume fractions of the interface components and the thickness of the interface layer. The simulation shows that the interface layer is modeled as a three-component $\mathrm{GaAs} / \mathrm{GaN} /$ voids composite whose composition varies with time, i.e., the $\mathrm{GaN}$ volume fraction increases at the expense of GaAs until 100\% GaN is reached. Then, the GaN bulk 2D-growth starts, and the GaN layer thickness increases linearly with time at about 1 $\AA /$ sec. The voids content at the interface simulates either the presence of structural defects and the grain boundaries.

Figure 2 shows the experimental SE spectra, at room temperature, of the real, $\left\langle\varepsilon_{1}\right\rangle$, and imaginary, $\left\langle\varepsilon_{2}\right\rangle$ part of the pseudodielectric function of the GaN buffer layers recorded at the end of the growth. In the figure, the solid lines are for the fitting results according to the models shown in the inset. A two-layer model (substrate/bulk GaN/surface roughness) provides the best fit for the $\mathrm{SE}$ spectrum of $\mathrm{GaN}$ grown on $\alpha-\mathrm{Al}_{2} \mathrm{O}_{3}$. The surface roughness developed during the growth is simulated by an outer layer which is a mixture of $\mathrm{GaN}+$ voids as depicted in fig. 2 .
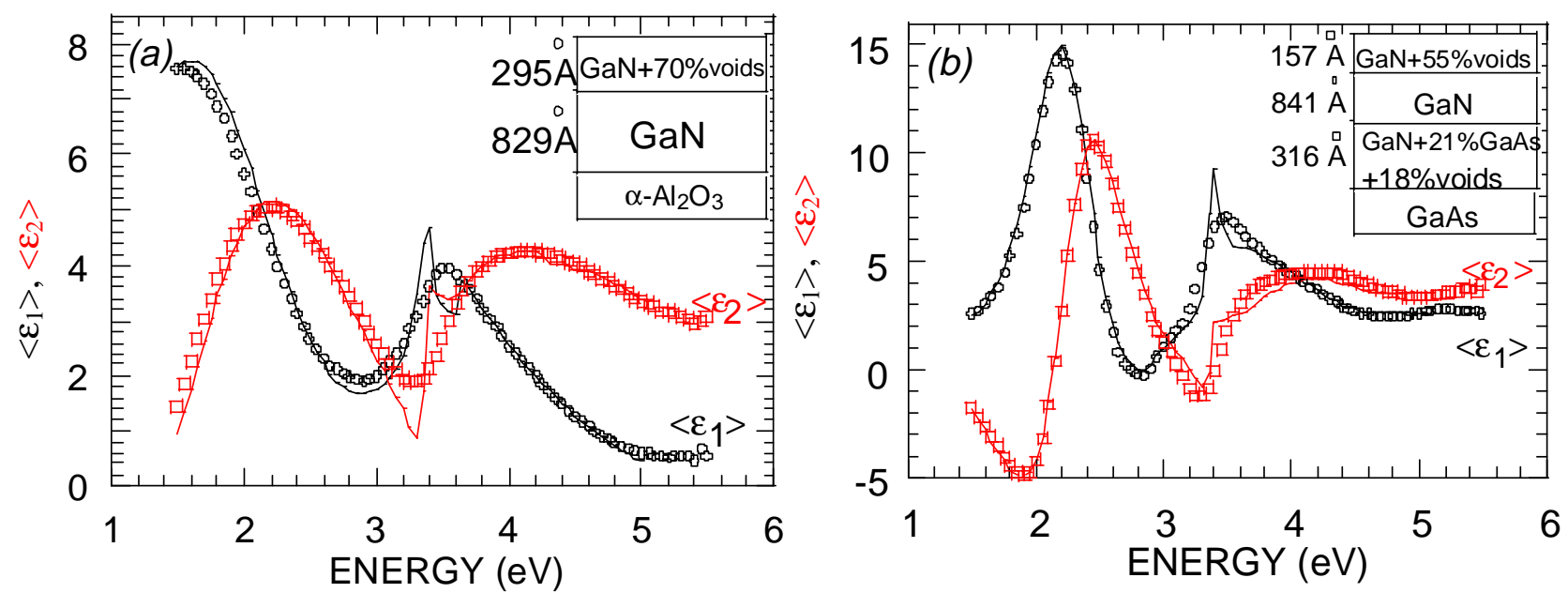

FIGURE 2. Real, $\left\langle\varepsilon_{1}\right\rangle$, and imaginary, $\left\langle\varepsilon_{2}\right\rangle$ part of the pseudodielectric function of the GaN layers grown on (a) $\alpha-\mathrm{Al}_{2} \mathrm{O}_{3}$ and (b) GaAs. The best-fit curves (full line) by the BEMA models shown in the inset are also reported. The standard deviations are of $\pm 1 \%$ on constituents volume fraction and of $\pm 10 \%$ on layer thickness. 
The best-fit model for the SE spectrum of GaN on GaAs employs a three-layer model (substrate/interface/bulk GaN/surface roughness) that accounts for the growth dynamics of fig. 1b: at first the interface formation between GaAs substrate and GaN film, and then the bulk GaN film growth. The evolution of the interface composition is approximated in the SE model by an average value (BEMA mixture of $\mathrm{GaN}+21 \% \mathrm{GaAs}+18 \%$ voids). The comparison of the fit and experimental data in both $\mathrm{GaN} / \alpha-\mathrm{Al}_{2} \mathrm{O}_{3}$ and $\mathrm{GaN} / \mathrm{GaAs}$ evidence the absence of a sharp excitonic transition, so indicating a very defective $\mathrm{GaN}$ buffer layer. Interestingly, cathodluminescence (CL) measurements have shown a very low intensity of the near-band edge emission and a well evident deep level emission at around $550 \mathrm{~nm}$. Also, AFM images have shown a grain-like morphology with an average diameter of $\sim 500 \AA$. These features have already been described by Tarsa et al.[8] and, they are, accordingly, related to the growth dynamics under N-stable conditions.

Thus, the amount of $\mathrm{N}$-atoms impinging on the growing films is a crucial parameter for the GaN growth. In the present RP-MOCVD apparatus, the performance of the nitrogen remote plasma source has been evaluated by OES measurements, performed in the afterglow at the substrate position. There, the OES spectra are dominated by the $\mathrm{N}_{2} 1$ st positive system [7], which indicates a considerable amount of $\mathrm{N}$-atoms and of electronically and vibrationally excited $\mathrm{N}_{2}$ molecules impinging on the growth surface. Any contribute of ions is absent and the importance of this can be understood by considering that the ionized portion of the $\mathrm{N}_{2}$ plasma beam has been suggested to have detrimental effect on the GaN film morphology and crystallinity [9].

Figure 3 shows the XRD measurements for GaN on both $\alpha-\mathrm{Al}_{2} \mathrm{O}_{3}(0001)$ and GaAs (001) substrates from which the crystallographic structure has been derived. The XRD pattern of GaN grown on GaAs shows only the (002) and (004) reflections, so demonstrating this film to be purely cubic and (001) oriented monocrystalline. The FWHM of the (002) reflection is 22 min for a film $\sim 100 \mathrm{~nm}$ thick, and this FWHM value is, to our knowledge, among the narrowest reported so far $[10,11]$. The $\mathrm{GaN}(002)$ reflection at $39.95^{\circ}$ corresponds to the $\mathrm{GaN}$ with a lattice constant of $4.509 \AA$, which is in good agreement with the calculated value of $4.503 \AA$ [12]. Also, from $\mathrm{XRD}$, there is no evidence of a ternary alloy $\mathrm{GaAs}_{\mathrm{x}} \mathrm{N}_{1-\mathrm{x}}$ at the interface, and a detailed analysis of the (002) peak shape, which is sensitive to compositional disorder, suggests that, indeed, phase separation of GaAs and GaN [11] does seem to occur at the interface, in agreement with the ellipsometric data. Here, it is important to underline that, in the present case, the pure cubic phase is grown without arsenic background pressure in the initial stage as reported by others $[10,11,13]$.
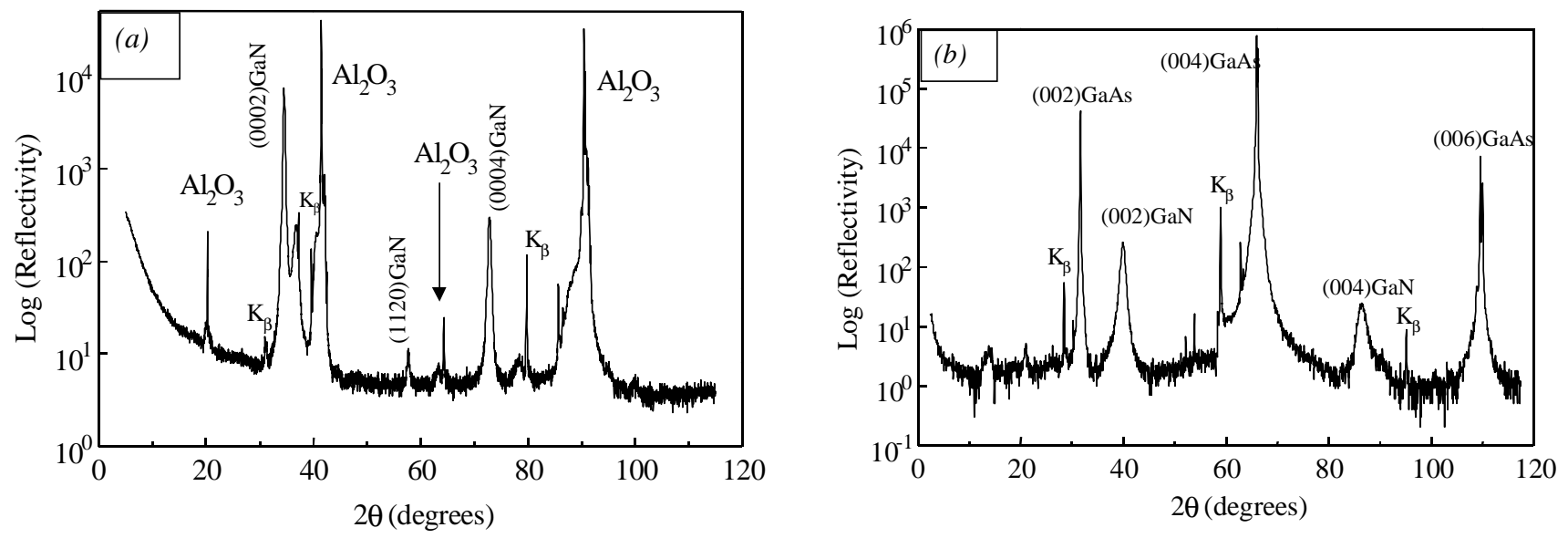

FIGURE 3. Typical XRD patterns of (a) GaN/ $\alpha-\mathrm{Al}_{2} \mathrm{O}_{3}$ (0001) and (b) GaN/GaAs(001) samples. 
In the present case, we have grown pure cubic GaN on a plasma nitrided GaAs surface differently from other workers $[14,15]$ who obtained hexagonal GaN on nitrided GaAs. This capability of our method is probably related to the fact that the remote plasma nitridation of the GaAs substrate occurs in absence of ion bombardment and under controlled conditions, so giving a very smooth ( RMS = 3.1 $\AA$ ) and homogeneous nitrided layer of $130 \AA$ in thickness [7,16], which acts as a template for the subsequent growth of the cubic phase. However, from the SE analysis of the $\mathrm{GaN}$ layer grown at $600^{\circ} \mathrm{C}$ (see model in fig. 2b), there is no memory of the layer coming from the pre-nitridation of the GaAs surface at $250^{\circ} \mathrm{C}$, i.e., the nitrided layer can not be discriminate after growth. This is, probably, because of its recrystallization and partial decomposition of the underlying GaAs surface during rise to growth temperature of $600^{\circ} \mathrm{C}$, thus resulting in the "new" $316 \AA$ interface layer of $(\mathrm{GaN}+21 \% \mathrm{GaAs}+18 \%$ voids $)$.

The XRD pattern of $\mathrm{GaN}$ grown on the (0001) plane of $\alpha-\mathrm{Al}_{2} \mathrm{O}_{3}$ (fig. 3a) shows the main peak at $34.55^{\circ}$ corresponding to the (0002) reflection from wurtzite $\mathrm{GaN}$ with a lattice constant in the c-direction of $5.18 \AA$ [12]. This confirms that the film is grown with its c-plane parallel to the substrate. The (0002) peak has a FWHM value of 8.6 min, which is well comparable with the best value of $\sim 5$ min reported in literature [17]. The feasibility of our system for the deposition of $\mathrm{GaN}$ buffer layer is the consequence of the $\alpha-\mathrm{Al}_{2} \mathrm{O}_{3}$ nitridation which has been performed under real time control by ellipsometry. In fact, $\alpha-\mathrm{Al}_{2} \mathrm{O}_{3}$ nitridation leads to different surface chemistry and morphology depending on exposure time [18,19]. Figure 4 shows the time evolution of the $\left\langle\varepsilon_{2}\right\rangle$ value during $\alpha-\mathrm{Al}_{2} \mathrm{O}_{3}$ nitridation. The decrease of $\left\langle\varepsilon_{2}\right\rangle$ is indicative of nitrogen incorporation, and the SE spectrum (spectrum B) recorded at the $\left\langle\varepsilon_{2}\right\rangle$ minimum can be modeled by a thin $(\sim 7 \AA)$ layer of a BEMA mixture of $\mathrm{AlN}+10 \% \mathrm{Al}_{2} \mathrm{O}_{3}$ which, however, can optically simulate an $\mathrm{AlNO}_{\mathrm{x}}$ layer also. AFM measurements of this $\mathrm{Al}_{2} \mathrm{O}_{3}$ nitrided surface show an atomically flat surface with a RMS value of $1.6 \AA$. For longer exposure time $(>10 \mathrm{~min}$ in the figure), the $\left\langle\varepsilon_{2}\right\rangle$ trend reverses as a consequence of roughening and of free aluminum formation, this last also confirmed by XPS measurements. The formation of free Al, besides roughening [19], could be the reason why long nitridation time results in a degradation of $\mathrm{GaN}$ layer quality $[18,19]$.
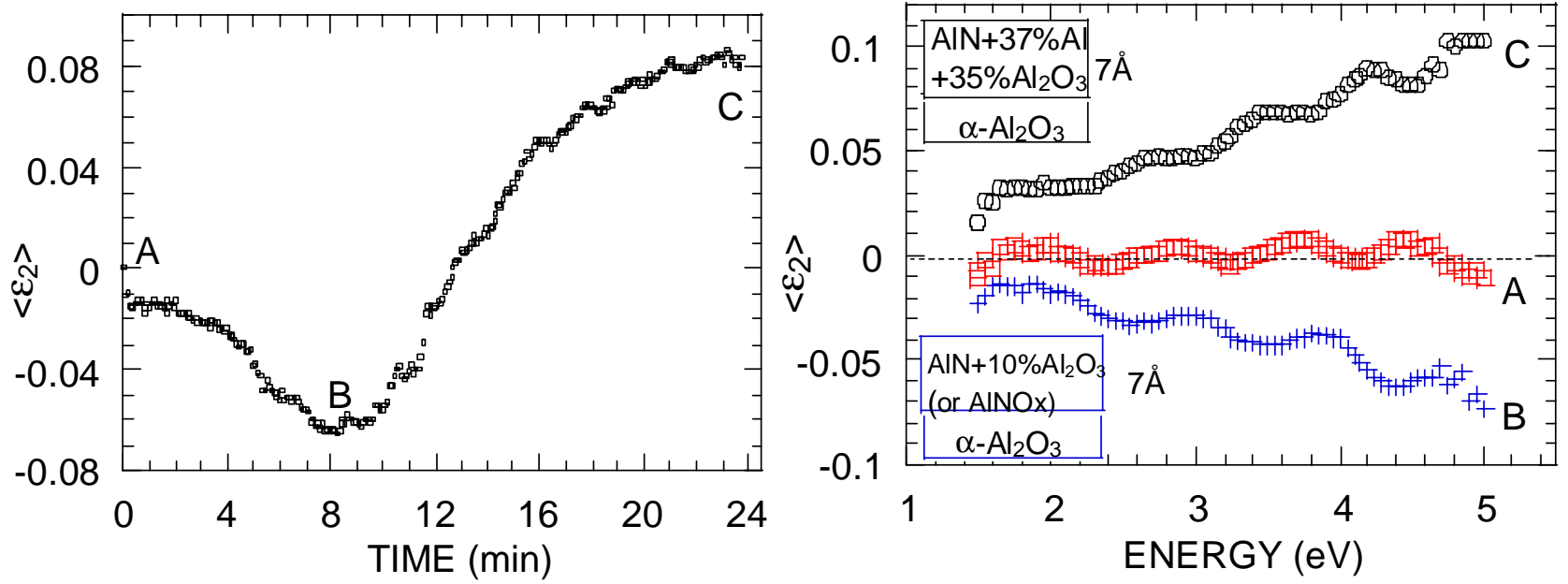

FIGURE 4. Time evolution of the $\left\langle\varepsilon_{2}\right\rangle$ value at $4.5 \mathrm{eV}$ during $\alpha-\mathrm{Al}_{2} \mathrm{O}_{3}$ nitridation (on the left) and SE spectra (on the right) at significant points of (A) $\mathrm{H}_{2}$ plasma cleaned $\alpha-\mathrm{Al}_{2} \mathrm{O}_{3}$ surface, (B) 8 min nitrided $\alpha-\mathrm{Al}_{2} \mathrm{O}_{3}$ surface, and (C) $25 \mathrm{~min}$ nitrided and damaged $\alpha-\mathrm{Al}_{2} \mathrm{O}_{3}$. The inset shows the best-fit BEMA models of spectra $\mathrm{B}$ and $\mathrm{C}$. 
Thus, the real time control by SE of the $\alpha-\mathrm{Al}_{2} \mathrm{O}_{3}$ nitridation has allowed the GaN growth to start soon after the minimum in $\left\langle\varepsilon_{2}\right\rangle$ (end-point of nitridation) has been reached.

\section{CONCLUSIONS}

In conclusion, spectroscopic ellipsometry has been applied as a real-time probe for the remote plasma MOCVD of GaN film in order to understand and optimize GaN growth and substrates nitridation processes. Ellipsometry has been shown to provide information on the endpoint of the substrate $\left(\mathrm{GaAs}\right.$ and $\left.\alpha-\mathrm{Al}_{2} \mathrm{O}_{3}\right)$ nitridation, on the substrate/GaN film interface and on the growth kinetics. The RP-MOCVD growth of $\mathrm{GaN}$ under $\mathrm{N}_{2}$ remote plasma preactivation conditions results in cubic $\mathrm{GaN}$ on $\mathrm{GaAs}$ and in hexagonal $\mathrm{GaN}$ on $\alpha-\mathrm{Al}_{2} \mathrm{O}_{3}$.

\section{ACKNOWLEDGEMENT}

Financial support from Progetto Finalizzato Materiali e Dispositivi per Elettronica a Stato Solido (MADESS) of the National Council of Research (CNR) is acknowledged.

\section{REFERENCES}

[1] S. Nakamura, Jpn. J. Appl. Phys. 30, L1705 (1991).

[2] T.D. Moustakas, T. Lei, R.J. Molnar, Physica B, 185, 36 (1993).

[3] K. Wang, D. Pavlidis, J. Singh, J. Appl. Phys. 80, 1823 (1996).

[4] B. Daudin, F. Widmann, G. Feuillet, Y. Samson, M. Arlery, J.L. Rouviere, Phys. Rev. B, 56, R7069 (1997).

[5] G. Bruno, P. Capezzuto, M. Losurdo, Phys. Rev. B, 54, 17175 (1996).

[6] D.E. Aspnes, J. Phys. (Paris) Colloq. 10, 3 (1983)

[7] M. Losurdo, P. Capezzuto, G. Bruno, E.A. Irene, Phys. Rev. B, 58, 1 (1998).

[8] E.J. Tarsa, B. Heying, X.H. Wu, P. Fini, S.P. DenBaars, J.S. Speck, J. Appl. Phys. 82, $5472(1997)$

[9] S. Gwo, H. Tokumoto, S. Miwa, Appl. Phys. Lett. 71, 362 (1997).

[10] H. Yang, O. Brandt, M. Wassermeier, J. Behrend, H.P. Schonherr, K.H. Ploog, Appl. Phys. Lett., 68, 244 (1996).

[11] T.S. Cheng, L.C. Jenkins, S.E. Hooper, C.T. Foxon, J.W. Orton, D.E. Lacklison, Appl. Phys. Lett., 66, 1509 (1995).

[12] J.H. Edgar, Properties of Group III Nitrides, EMIS Datareviews Series No. 11, INSPEC, London, United Kingdom, 1994.

[13] O. Brandt, H. Yang, B. Jenichen, Y. Suzuki, L. Daweritz, K.H. Ploog, Phys. Rev. B, 52, R2253 (1992).

[14] A. Kikuchi, H. Hoshi, K. Kishino, Jpn. J. Appl. Phys. 33, 688 (1994).

[15] O. Brandt, H. Yang, A. Trampet, M. Wassermeier, K.H. Ploog, Appl. Phys. Lett, 71, 473 (1997).

[16] M. Losurdo, P. Capezzuto, G. Bruno, P.R. Lefebvre, E.A. Irene, J. Vac. Sci. Technol. B, 16, 2665 (1998).

[17] O. Ambacher, J. Phys. D: Appl. Phys. 31, 2653 (1998).

[18] S. Keller, B.P. Keller, Y.F. Wu, B. Heying, D. Kapolnek, J.S. Speck, U.K. Mishra, S.P. DenBaars, Appl. Phys. Lett. 68, 1525 (1996)

[19] K. Uchida, A. Watanabe, F. Yano, M. Kouguchi, T.Tanaka, S. Minagawa, J. Appl. Phys. 79, 3487 (1996). 